\title{
LA NATURALEZA DEL PLACER EN EL FILEBO DE PLATÓN
}

Francisco Bravo*

\begin{abstract}
RESUMEN
Examino, en esta comunicación, la respuesta de Platón a una de las cuestiones fundamentales sobre el placer, legada por la tradición griega didáctica: héntiva physin échei, ¿Qué es? ¿Cuál es su naturaleza? Lo hago sólo desde los puntos de vista físico y fisiológico, dejando de lado los aspectos psicológico, epistemológico y ontológico. En el primero de estos planos, Platón concibe el placer como cierto tipo de movimiento (kinêsis tis). En el segundo, como un movimiento de repleción (plêrôsis). ¿Qué debe entenderse por estos términos definicionales. En particular, ¿abarca la plêrôsis hedónica tanto la repleción en tanto proceso (in fieri) -el proceso de llenarse- como la repleción en tanto resultado (ut factum esse) el hecho de estar lleno, la plenitud- o sólo la primera? Mi investigación se desarrolla retomando a posteriori la interpretación del neoplatónico Damascio y en diálogo con el filósofo norteamericano George Rudebusch.
\end{abstract}

TÉRMINOS CLAVE: Placer. Naturaleza. Movimiento. Repleción.

\begin{abstract}
My aim in this article is to examine the Plato's answer to one of the essencial questions about pleasure, laid down by greek didactic tradition: héntina physin échei; What is it? What is its nature? I'm

\footnotetext{
* Professor Titular do Departamento de Filosofia da Universidade Central da Venezuela e membro do Instituto Internacional de Filosofia. E-mail: fbravovi@yahoo.com.br
} 
going to deal only with the physical and physiological point of view, leaving behind the aspects concerning psychology, epistemology and ontology. On the first case, Plato conceived the pleasure as some kind of movement (kinesis tis). On the second, as a movement of contentment (plêrosis). What should we understand about this terms? Does the hedonic plêrosis embrace as the contentment as the process (in fieri) of become contented?

My investigation goes on retaking the interpretation of Damascio and a dialoghe with the North-American philosopher George Rudebusch.

KEY-WORDS: Pleasure. Nature. Movement. Contentment.

\section{Planteamiento y evolución del problema}

Una de las preguntas sobre el placer que la tradición "didáctica" legó a Platón es la concerniente a su naturaleza: ¿qué es el placer? Ella equivale a preguntar cuáles son las condiciones -físicas, fisiológicas, psicológicas, ontológicas- de su realización?

Esta pregunta se halla en ciernes desde el Protágoras, considerado de ordinario como un diálogo de juventud. Este diálogo, (1) maneja varios términos hedónicos, con diversos matices de significación (cf: 337c); (2) sostiene que todo placer tiene un objeto determinado (cf. 351c2); (3) reconoce una causa del mismo, que no es otra que $\tau \dot{\alpha} \hat{\eta} \delta \delta \varepsilon \alpha$, las cosas agradables (cf. 351e1). En el Gorgias, probablemente contemporáneo del Protágoras, Platón empieza a explicitar el problema de la naturaleza del placer cuando sostiene que las $\varepsilon \mu \pi \varepsilon ı p^{\prime} \alpha$ o prácticas rutinarias como la culinaria

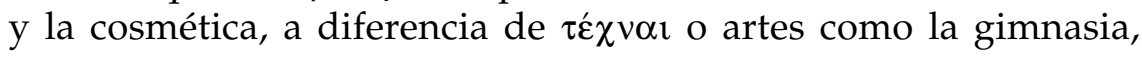

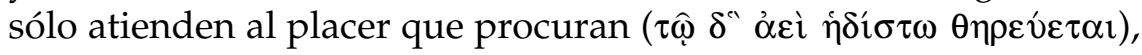

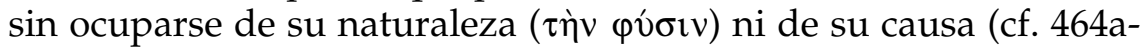
66a; 501a5-6). Además, introduce el término $\pi \lambda \eta \dot{\rho} \omega \sigma \iota \varsigma$ (repleción), que terminará siendo el definiens del placer desde el punto de vista fisiológico (cf. 492b1, 496e2). En fin, en este diálogo empieza a abordar el problema de la relación entre el placer y el dolor (cf.

Educ. e Filos., Uberlândia, v. 22, n. 43, p. 139-160, jan./jun. 2008 
494e-497d), que se agudizará en el Fedón (60b-c), la República (584b3, c1-2) y el Filebo (31b5-6). El Hipias Mayor relaciona el problema de la naturaleza del placer con el de la naturaleza de lo bello (cf. 297e-300c) y plantea, en este contexto, esta pregunta netamente definicional: " $¿$ difiere un placer de otro placer en tanto placer?" (299d2-3). No se trata de saber si un placer es más grande o menos grande y si hay en él el más o el menos, sino "si la diferencia entre placeres en tanto placeres está en que uno de ellos es un placer y el otro no lo es" (299d4-5). ¿Qué es, pues, lo que hace que un placer sea un placer?

La contribución de República a la solución de este problema es decisiva, pues pone uno y confirma otro de los elementos definitorios del placer: primero, desde el punto de vista físico,

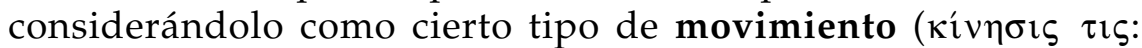
583e10); segundo, desde el punto de vista fisiológico, concibiéndolo, lo mismo que el Gorgias, como un movimiento de repleción ( $\pi \lambda \eta \dot{\rho} \rho \omega$ เৎ: 585a3; cf. 585b9, 439d8). Más aún, generaliza estos dos elementos y los extiende tanto a los placeres del cuerpo como a los puramente mentales (585a-b). Pero es el Filebo -este diálogo que

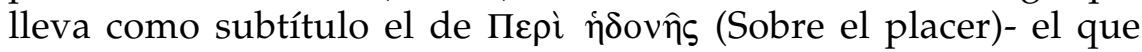
aborda sistemáticamente el problema de la naturaleza del placer. Cree el autor que, siendo así que, en el debate sobre el Bien Supremo del hombre, una de las posturas es la que lo identifica con el placer,

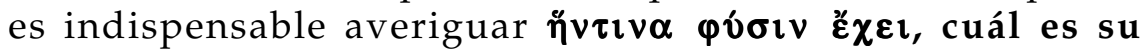
naturaleza (12a4-6). Responderá, desde el punto de vista

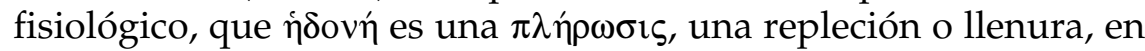

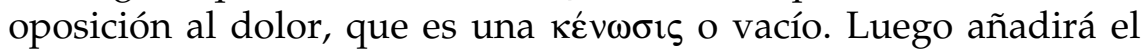
elemento que lo define desde el punto de vista psicológico, concibiendo los placeres como $\pi \lambda \eta \rho \omega ́ \sigma \varepsilon \imath \varsigma \alpha i \sigma \theta \eta \tau \alpha i$, como repleciones sentidas (51b6). Más aún, defínelo en un plano netamente ontológico, incluyéndolo indirectamente en el género de lo Ilimitado (ő $\pi \varepsilon ı \rho \rho v: ~ 23 c 9)$, y directamente en el género de lo Mixto de Ilimitado y Límite (23d1).

Analizaré a continuación los aspectos físico y fisiológico del placer, teniendo presentes las observaciones de mis críticos, especialmente del filósofo norteamericano George Rudebusch, a 
las posturas que he defendido en mi libro Las ambigüedades del placer. Ensayo sobre el placer en la filosofía de Platón (Sankt Augustin, Academia Verlag, 2003).

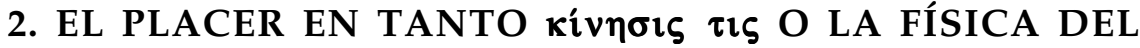 PLACER}

En oposición a ciertos pensadores contemporáneos, que concebían el placer como una especie de reposo ${ }^{1}$, Platón sostiene que, por producirse en el alma ( $\dot{\varepsilon} v \psi v \chi \hat{\eta} \gamma(\gamma v o ́ \mu \varepsilon v o v)$, éste es cierto

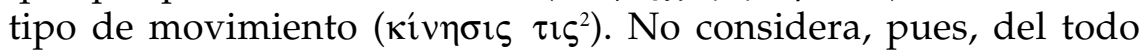
extraña la opinión de algunos $\kappa{ }^{\prime} \mu$ o $^{3}$ (sutiles) que sostienen que

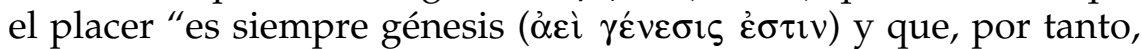

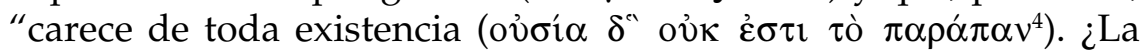
acepta teóricamente? ¿Sostiene que íjovń es génesis y nada más que génesis? ${ }^{5}$ Mi punto de vista, en el que ahondaré más adelante,

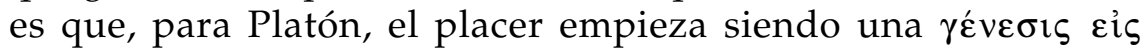
ov̉ $\sigma^{\prime} \alpha v^{6}$ (una generación camino de la existencia) y termina siendo, una vez que ha cumplido con ciertos requisitos, una $\gamma \varepsilon \gamma \varepsilon v \eta \mu \varepsilon ́ v \eta$ ov̉ $\mathbf{i}^{7} \alpha^{7}$, una existencia generada.

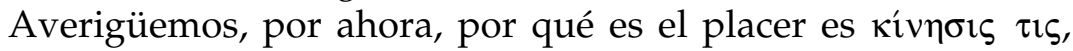
cierto tipo de movimiento. En República, la respuesta parece darse en la frase ' $\varepsilon v \psi v \chi \hat{n} \gamma \imath \gamma \nu o ́ \mu \varepsilon v o v$ ', que precede a la enunciación de la tesis del placer-movimiento. Pero esta frase puede traducirse de dos modos: (1) "cuando se produce en el alma", como la traduce E. Chambry", entre otros; (2) "por producirse en el alma". Creo que la primera traducción es inaceptable, pues da a entender que

1 Cf. Rep. 583c-584 y Fil. 43d ss.

2 Rep. IX, 583e10.

3 Fil. 53c6.

4 Fil. 53 c 5.

5 Cf. Bravo (2003) 61.

6 Fil. 26 d 9.

7 Fil. 27 b 8.

8 Platon, Republique, Paris, Les Belles Lettres, col. G. Budé, 1973.

Educ. e Filos., Uberlândia, v. 22, n. 43, p. 139-160, jan./jun. 2008. 
algunos placeres no se producen en el alma, lo cual se opone a una tesis a la que Platón llega en el Filebo como resultado de una larga evolución": la tesis según la cual "el apetito, el deseo, el principio de todo animal pertenece al alma" (35d1-2). Sólo la segunda

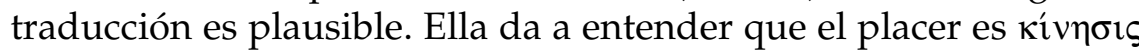
$\tau \iota \varsigma$ porque se produce en el alma, que, en sí y por sí, es movimiento y principio de todo movimiento. No pueden ser otra cosa los estados que en ella tienen lugar. Pero esto parece implicar, o bien que todos los placeres son mentales (a), o bien que la tesis del placermovimiento se aplica únicamente a los placeres mentales (b). Es claro que (a) se opone a la división de los placeres en somáticos y mentales, sostenida a lo largo de todo el Filebo, particularmente en 31e-32c. En cuanto a (b), desconoce el rechazo de Platón a la tesis del placer-reposo: según el autor, por ser un simple reposo y por tanto una ausencia de movimiento, los pretendidos placeres-reposo (de un dolor), lo mismo que los dolores-reposo (de un placer) constituyen la clase más falsa de los placeres falsos: "la ausencia del dolor y el placer -dice Sócrates- son distintos por naturaleza" $\left(44^{\mathrm{a}} 11\right)$.

Podemos, pues, concluir que el placer es cierto tipo de movimiento porque su sujeto último es el alma, que es movimiento

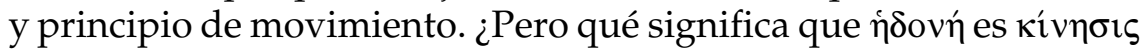
$\tau ı \varsigma$ ? ¿Quiere esta tesis decir que el movimiento es un elemento del placer, o más bien que es la causa eficiente del mismo? La respuesta del Timeo parece ser la segunda, aunque refiriéndose directamente tan sólo a los placeres corpóreos: la causa de éstos son ciertos $\pi \alpha \theta \eta \dot{\eta} \mu \alpha \alpha$, ciertas impresiones o movimientos producidos en el cuerpo $^{10}$. Unos movimientos que deben cumplir con ciertas condiciones. La primera de ellas es que se produzcan en una parte fácilmente movible del cuerpo, a saber, en una parte compuesta mayormente de aire y fuego: "si una cosa es por naturaleza muy

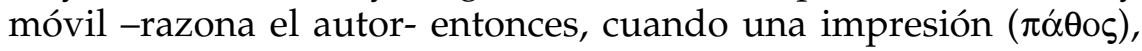

9 Cf. Bravo (2003) 85.

${ }^{10}$ Cf. Tim. 64 a 3. 
incluso muy breve, actúa sobre ella, sus diferentes partes se transmiten en círculo" (64b3-4) y llegan a "la parte inteligente del alma" (64b6), que es la sede de la conciencia. Cuando esto ocurre,

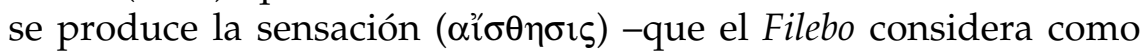

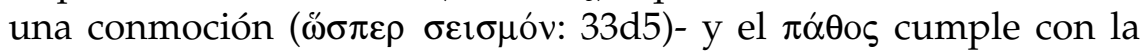
segunda condición para ser causa del placer o del dolor, a saber,

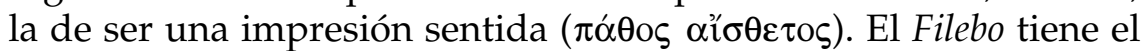
cuidado de señalar que esto no siempre ocurre: que algunas afecciones del cuerpo "se apagan en el cuerpo antes de llegar al alma y dejan a ésta inafectada ( $\dot{\alpha} \pi \alpha \hat{\eta})$ " (33d4). Pero incluso si ocurre y la sensación se produce, la impresión sentida debe cumplir con una nueva condición: debe ser brusca ( $\dot{\alpha} \theta \rho o ́ o v: ~ 64 \mathrm{~d} 1$ ), pues la que se produce paulatinamente $\left(\mu \varepsilon \tau^{\prime} \varepsilon \hat{v} \pi \varepsilon \tau \varepsilon \dot{i} \alpha \varsigma\right)$, es ciertamente sensible, pero no comporta ni placer ni dolor $(64 \mathrm{~d} 3-5)^{11}$. En fin, la impresión bruscamente sentida debe ser tal que restablezca el estado

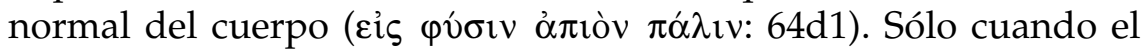
movimiento corpóreo se produce en una parte fácilmente movible del cuerpo, tiene lugar bruscamente y restablece el estado natural del cuerpo, se transforma en una sensación y en una sensación agradable, es decir, en un placer propiamente dicho. Aclaremos

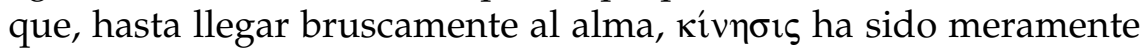
corpórea, y, como tal, ha actuado como causa eficiente del placer, manteniéndose exterior a él al igual que toda causa eficiente. Pero cuando, tras $\varepsilon \lambda \sigma \varepsilon i ́ \sigma \mu o s$, se transforma en un movimiento del alma, pasa a ser, por el mismo hecho, elemento constitutivo y ratio formalis del placer somático. Tratándose del placer puramente mental -por ejemplo, de los placeres epistémicos ${ }^{12}$, generados en el alma y situados en ella- el movimiento es aún con más claridad, no sólo su causa eficiente, sino también su causa formal, pues nace en el alma y es parte del alma, que es movimiento y causa del movimiento. Este breve análisis muestra que, para entender la naturaleza del placer, hay que ahondar en la doctrina platónica

${ }^{11}$ Cf. A.E. Taylor (1928) 448.

${ }^{12}$ Cf. Fil. 51 c.

Educ. e Filos., Uberlândia, v. 22, n. 43, p. 139-160, jan./jun. 2008. 
del movimiento ${ }^{13}$. ¿Qué tipo de movimiento es el placer? Limitémonos a decir que, tras una larga evolución, imposible de recorrer en el marco de esta charla, Platón puede afirmar, primero, en la República (485d11,580d y 581c), luego en el Filebo (35b11) y finalmente en las Leyes ${ }^{14}$, que $\dot{\eta} \delta$ oví es cierto movimiento del alma, incluso cuando parece tener lugar únicamente en el cuerpo.

\section{EL PLACER EN TANTO PLHRWSIS O LA FISIOLOGÍA DEL PLACER}

Queda por especificar qué clase de movimiento mental es el placer. La respuesta del Filebo viene dada por la tesis del placerrepleción, y constituye lo que podríamos llamar la fisiología del placer. Recordemos que 'fisiología' y 'fisiológico' designan ordinariamente funciones de los cuerpos vivientes; pero a veces también se aplican, por extensión, al estudio de las funciones mentales ${ }^{15}$. Nos valdremos, pues, del uso secundario de estos

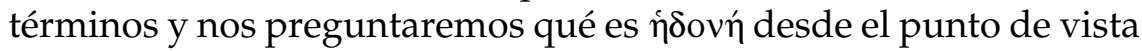
fisiológico.

\subsection{La definición fisiológica del placer}

Como vimos, la respuesta a esta pregunta empieza a darse desde el Gorgias, se generaliza en República IX y alcanza su status definicional en el Filebo, y lo hace en. En el primero de estos diálogos, Calicles, pensando única o preferentemente en el placer somático, lo concibe como una $\pi \lambda \eta \dot{\rho} \omega \sigma \iota \varsigma$ o repleción en sentido activo, es decir, como un proceso de repleción en el cuerpo. Según este personaje, representativo de cierta tendencia de la época, el placer se da sólo mientras dura este proceso, pues "para el hombre ya

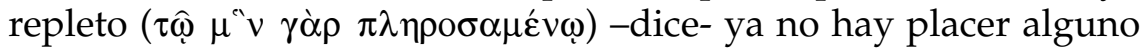

${ }^{13}$ Cf. Bravo (2003) 47-54.

${ }^{14}$ Bravo (2003) 54.

${ }^{15}$ Cf., por ejemplo, Maudsley, Physiology and Pathology of Mind, 1867, cit. por A. Lalande, Vocabulaire technique et critique de la philosophie, Paris, P.U.F, 1968, p. 780. 


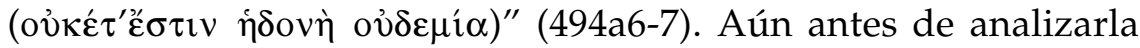
suficientemente, Sócrates tiende a aceptar la concepción del placer corporal como un proceso de repleción. El placer de beber cuando tenemos sed, por ejemplo, "es la repleción de una carencia

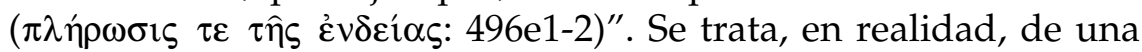
concepción pre-platónica, frecuente en el transcurso del siglo $\mathrm{V}$ y canonizada por Empédocles ${ }^{16}$ y el círculo pro-pitagórico del médico-filósofo Almeón de Crotona ${ }^{17}$. En República, esta concepción de $\dot{\eta} \delta$ oví se extiende a los placeres mentales y eo ipso al placer globalmente considerado. Después de recordar que el hambre, la sed y otros fenómenos de la misma índole son "tipos de vacíos en el estado del cuerpo", Platón añade que: (a) la ignorancia y la

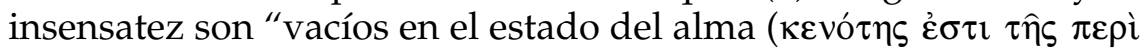

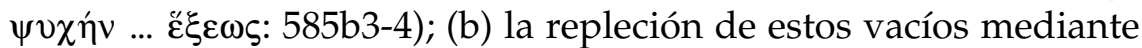
la instrucción y la educación constituyen placeres de la mente. En el Filebo, esta concepción fisiológica se funda en una fisiología más amplia, desarrollada entre las páginas 32c y $36 \mathrm{c}^{18}$

Para mejor entender la definición fisiológica del placer es necesario tener presente que, según la proposición ontológica fundamental del Filebo, "todo lo que actualmente existe en el

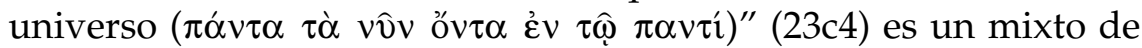
Ilimitado y Límite (cf. 23c, 16c10). El estado natural de este mixto es la armonía entre sus dos constitutivos esenciales (cf. 32a-b). Por eso, cuando "en nosotros los vivientes la armonía se disuelve

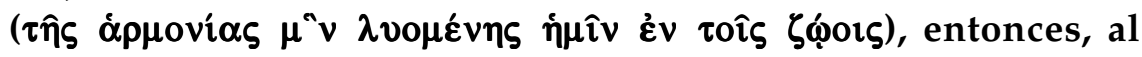
mismo tiempo que de este modo se disuelve la naturaleza ( $\alpha \mu \alpha$

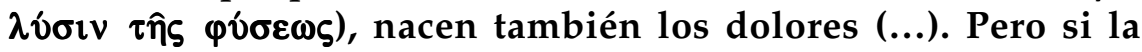
armonía se recompone y la naturaleza propia se reconstituye

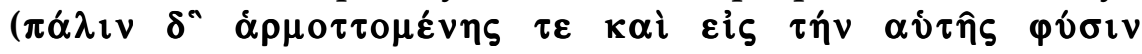

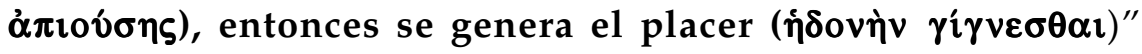

${ }^{16}$ Aecio 4, 9, 15 = DK A 95; cf. Bravo (2003) 56, nota 7.

${ }^{17}$ Cf. Aecio 5, 30, 1; Bravo (2003) 56, nota 9.

${ }^{18} \mathrm{Cf}$. Gerd van Riel, "Le plaisir est-il la répletion d'un manque? La définition du plaisir (32a-36c) et la physiologie des plaisirs faux (42c-44a)", in: M. Dixsaut, La fêlure du plaisir, Paris, J. Vrin, 1999, 301.

Educ. e Filos., Uberlândia, v. 22, n. 43, p. 139-160, jan./jun. 2008 
(31d4-9). Sócrates abunda en ejemplos tomados de la vida

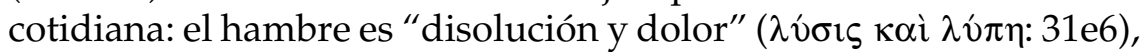
mientras que el alimento, que es repleción y plenitud recuperada

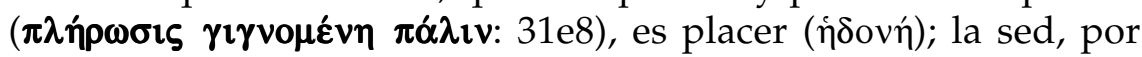
su parte, es trastorno y dolor, pero cuando el líquido vuelve a llenar $(\pi \lambda \eta \rho о v \hat{\sigma \alpha} \alpha)$ lo que estaba vacío, es placer (31e-32a). Cabe notar que, en este juego de relaciones, naturaleza, armonía, repleción y placer son hasta cierto punto correlativos. La naturaleza es armonía entre el Límite y lo Ilimitado; si esta armonía se destruye, la naturaleza se destruye igualmente; cuando ella se reconstruye, es decir, cuando hay una repleción del vacío dejado por su destrucción, hay un "retorno a la naturaleza" y "convenimos -

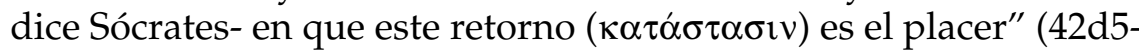
6). Diríamos entonces que la naturaleza no puede darse sin la armonía ni la armonía sin el placer. O a la inversa, el placer no puede darse sin el retorno a la naturaleza, que es como decir sin el retorno a la armonía. Por ello, sin duda, Afrodita -no la de Filebo, asociada con el placer sin regla ni medida (cf. Fil. 12c), sino la de Hesiódo $^{19}$, adoptada por Sócrates- es madre de la armonía, pues introduce "la ley y el orden, portadores del límite" (Fil., 26b). Insistamos: naturaleza humana bien lograda, armonía vital de ilimitado y límite y repleción son tres descripciones del placer que tienen el mismo valor, desde el punto de vista fisiológico.

La repleción $(\pi \lambda \eta \dot{\eta} \omega \sigma \iota \varsigma)$ es, en todo caso, la condición necesaria y suficiente de las otras dos. Ella constituye la diferencia específica del movimiento-placer ( $(\lambda v \pi \eta ́)$. En esta línea interpretativa se sitúa Aristóteles cuando dice que, para los platónicos, "el dolor es una deficiencia del estado

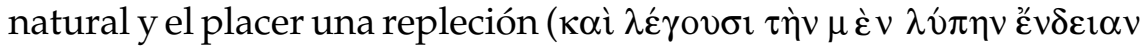

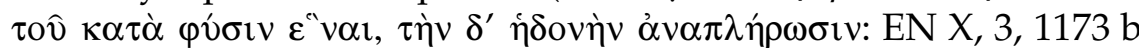
8-9). Damascio subraya, por su parte, la generalidad de este definiens, extendiéndolo igualmente al universo de los placeres puros

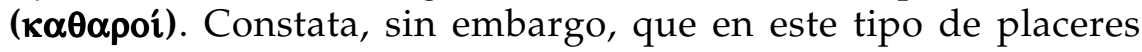

${ }^{19}$ Teog., 937, 975.

Educ. e Filos., Uberlândia, v. 22, n. 43, p. 139-160, jan.jun. 2008 
parece no haber una carencia previa, y pregunta "cómo entonces puede la percepción ser estimulada para buscar la repleción, de la

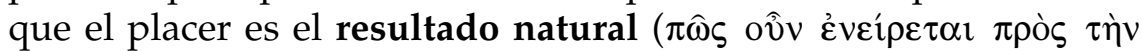

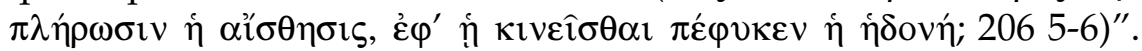
Su primera hipótesis es que, al no haber una deficiencia previa, la repleción parece innecesaria (206, 6-7). Pero luego explica que, en el caso de los placeres puros, la deficiencia previa, aunque existe realmente, lo mismo que en el de las otras clases de placeres, es

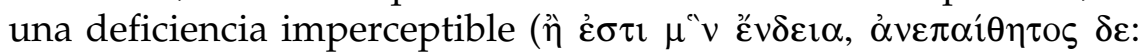
206, 7). Y precisa aún más: “cuando prevalece lo natural (es decir, el estado de bienestar), la repleción es procurada por algo que

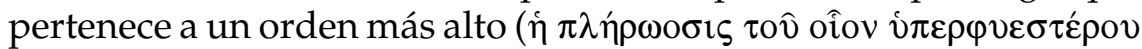

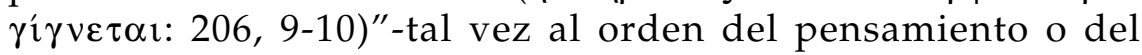
sentimiento; y el organismo requiere de ello, "no porque algo se ha

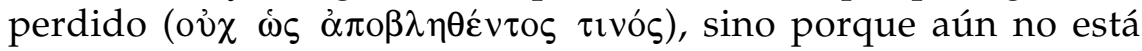

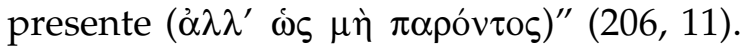

\subsection{El sentido de ' $\pi \lambda \eta \dot{\rho} \omega \sigma \iota \varsigma^{\prime}$}

¿Pero cómo debe entenderse el término ' $\pi \lambda \eta \dot{\rho} \omega \sigma \iota \varsigma^{\prime}$ en tanto definiens fisiológico del definiendum que nos ocupa? Empecemos recordando que este sustantivo se repite once veces en el Filebo: $31 \mathrm{e} 8$; 35a1, 2, 7; 35b4, 6, 11; 36b12; 42c10; 47c6; 51b6, mientras que el verbo correspondiente $(\pi \lambda \eta \rho \hat{\omega})$ ocurre seis veces: $32 \mathrm{a} 1 ; 35 \mathrm{a} 4$; 35e10; 36b1, 4; 52a5. ¿Qué significa, en estos textos, ' $\pi \lambda \eta \dot{\eta} \rho \omega \sigma \varsigma^{\prime}$ y sus cognados en tanto definiens de ídoví?

Hablando en general, el verbo $\pi \lambda \eta \rho \hat{\omega}$ es transitivo, con el significado de llenar o completar, o intransitivo, con el significado de estar lleno, completo o acabado. Según el diccionario de A. Bailly (Dictionnaire Grec-Français, 16e, 1950, p. 1573), este doble significado del verbo se transmite también al sustantivo, que significa: (a) en sentido activo, el proceso de llenar, la repleción propiamente dicha; (b) en sentido pasivo, el estado que resulta del proceso, el estado de estar lleno, es decir, la plenitud. El segundo de estos sentidos ha sido utilizado, entre otros, por A. Diès, que

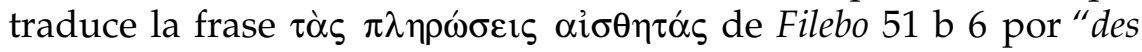

Educ. e Filos., Uberlândia, v. 22, n. 43, p. 139-160, jan./jun. 2008 
plénitudes senties"; y por E. Chambry, que traduce la frase $\pi \lambda \eta \dot{\eta} \rho \omega{ }^{\prime} \varsigma$ $\delta^{\prime \prime} \alpha \dot{\lambda} \eta \theta \varepsilon \dot{\sigma} \sigma \varepsilon \rho \alpha$ de República $585 \mathrm{~b} 9$ por "la plenitude la plus réelle". Pero aunque todos aceptan el sentido activo, no todos suscriben el pasivo. ¿Es plausible este segundo sentido en la definición fisiológica del placer dada por Filebo 31d ss? ¿Puede el placer entenderse, no sólo como el proceso de llenar un vacío previamente existente, sino también como un estado, la plenitud que resulta del proceso? Es obvio que la respuesta es importante, no sólo en el plano ontológico, sino sobre todo desde el punto de vista ético: si, como algunos pretenden, el placer no es más que génesis y no tiene existencia alguna ${ }^{20}$, entonces habrá que colocarlo en otra parte que en la clase del bien ${ }^{21}$

\subsubsection{La distinción de Damascio}

El doble sentido de $\pi \lambda \eta ́ \rho \omega \sigma \iota \varsigma$ fue defendido en la antigüedad por el neoplatónico Damascio (ca. 470), de la Escuela de Atenas,

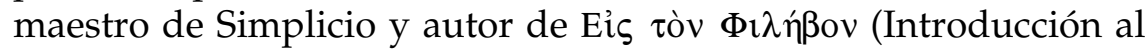
Filebo), el único comentario de este diálogo legado por los antiguos ${ }^{22}$. Damascio distingue, en la $\pi \lambda \eta ́ p \omega \sigma \iota \varsigma$ hedónica, una primera etapa, que llama proceso, y una segunda, resultado de la anterior, que

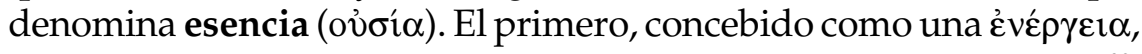

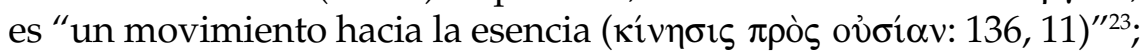
la segunda, a saber, la esencia, constituye la "condición natural ( $\tau$ ò

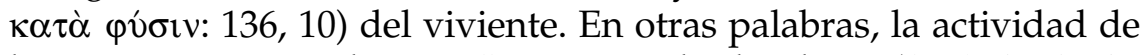

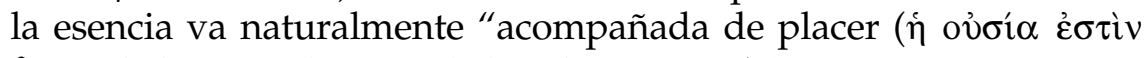

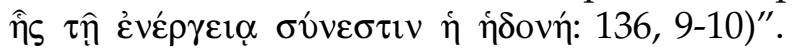

Hay, pues, según Damascio, dos clases de placeres, desde el punto de vista físico-fisiológico: el placer-en-movimiento, que se

${ }^{20}$ Cf. Fil. 53c.

${ }^{21}$ Fil. $54 \mathrm{~d}$.

${ }^{22}$ Hay ediciones de G. Stallbaum, Platonis Philebum, Lipsiae, 1820, y de L.G. Westerink, Lectures on the Philebus wrongly attributed to Olympiodorus, Amsterdam, North Holland, 1959.

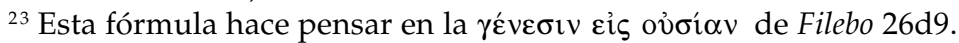


identifica con la repleción-proceso, y el placer-en-reposo, que se identifica con la repleción-resultado. El placer puro ( $\kappa \alpha \theta \alpha \rho \eta ́)$-único placer propiamente dicho, según el Filebo- puede darse en cualquiera de las dos clases. Lo propio de él es que el vacío que le precede es un vacío no-sentido: el viviente requiere de él, "no por haber perdido una cosa, sino porque ésta no le está aún presente

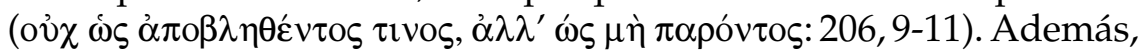
Damascio también identifica el placer en reposo - que constituye la "condición natural" del viviente- con el placer catastémico de

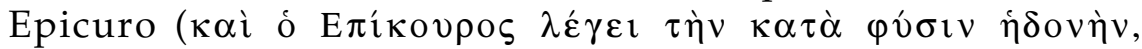

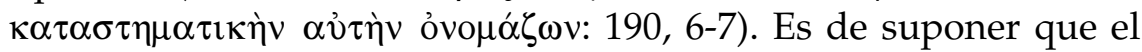
placer en movimiento se identifica con el cinético del Jefe del Jardín. En nuestra época, Donald Davidson parece acercarse a esta distinción cuando cree descubrir en el Filebo placeres con proceso y placeres sin proceso. Pero ésta es una distinción equívoca, pues incluso si admitimos la división propuesta por la interpretación de Damascio, no hay, para el Filebo, placeres sin proceso: también el placer-en-reposo se da como resultado de un proceso ${ }^{24}$; y también en este sentido, como dice Aristóteles, "no hay sólo una actividad del movimiento, sino también una actividad del reposo (ov̉ rò $\rho$

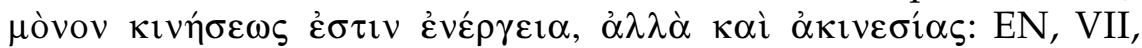
$1154 b 27-28)$. Damascio va más allá y concluye que "el paradigma de todo placer es el placer en reposo del intelecto". Su interpretación se aproxima a la postura de Aristóteles, según el

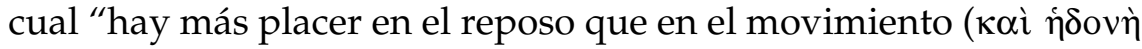

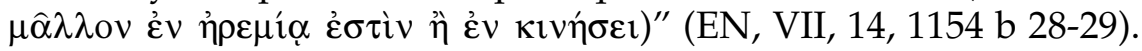
Parece claro que el placer-reposo no es actividad de llenar, sino estado de estar lleno, plenitud.

\subsubsection{Objeciones a la distinción entre placer-repleción y placer- plenitud.}

¿Es fiel a los textos del Filebo la distinción hecha por Damascio, que yo he defendido independientemente de él en mi libro Las

${ }^{24}$ Cf. Bravo (2003) 138.

Educ. e Filos., Uberlândia, v. 22, n. 43, p. 139-160, jan./jun. 2008 
ambigüedades del placer? Me limitaré a examinar las objeciones de G. Van Riel ${ }^{25}$ y G. Rudebusch.

1. Van Riel cree que la interpretación de Damascio representa una "modificación profunda" de la tesis platónica del placerrepleción. En particular, no ve en qué textos del Filebo podría fundarse la tesis de que el placer-en-reposo es una repleción. Rechaza, en consecuencia, la idea de una repleción-resultado, sinónimo de plenitud.

Creo, en contra de Van Riel, que la interpretación de Damascio es fiel a la letra y aún más al espíritu del Filebo. Según este diálogo,

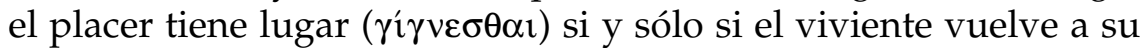

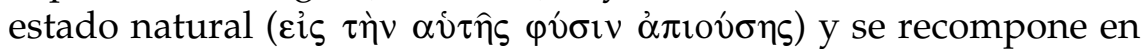

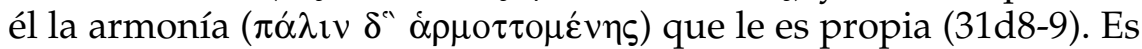

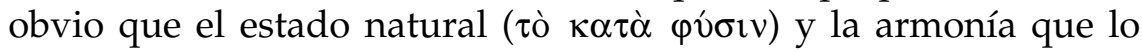
caracteriza son estados (๕̌ $\xi \varepsilon 1 \varsigma$ ) y no se confunden con los procesos ( $\gamma \varepsilon v \varepsilon \varepsilon \sigma \varepsilon i \varsigma)$ que los originan. Así lo indican además: (1) en el dominio

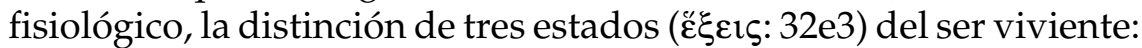
uno de destrucción de su estado natural, otro de restauración del

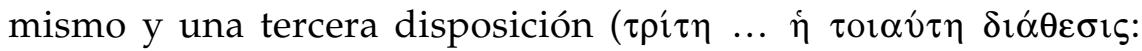
32e9) en que no hay ni destrucción ni restauración (32e); (2) en el dominio ético, el propósito de los interlocutores de buscar "una

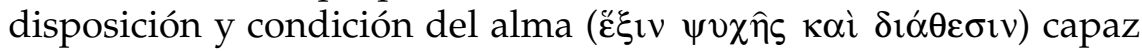
de asegurar a todos los hombres la vida dichosa"26. No es plausible que una disposición y una condición del ser viviente posea, entre sus elementos, un simple proceso. Y esto nos lleva a concluir que la

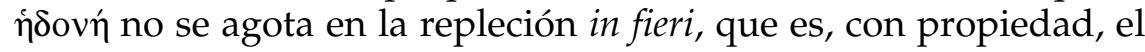
camino hacia ella, sino que abarca también la repleción como factum esse, es decir, como disposición o estado. En virtud de ello puede constituir, no exclusivamente pero sí inclusivamente, juntamente con el intelecto, el "mixto" que es la vida buena, la cual, por ser el bien supremo del hombre, no es un simple proceso, sino un estado

${ }^{25}$ G. Van Riel, “Le plaisir est-il la repletion d'un manque? La définition du plaisir (32a-36c) et la phisiologie des plaisirs faux", in: M. Dixsaut (Ed.), La fêlure du plaisir, Paris, J. Vrin, 1999, pp. 299-314.

${ }^{26}$ Fil. $11 \mathrm{~d} 4-6$.

Educ. e Filos., Uberlândia, v. 22, n. 43, p. 139-160, jan./jun. 2008 
constituido. Insistimos: no puede ocurrir que uno de los componentes de este estado sea un simple proceso. Según Damascio, la $\pi \lambda \eta \dot{\eta} \omega \sigma \mathrm{is}$ en tanto proceso es, hablando con

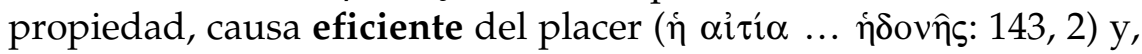

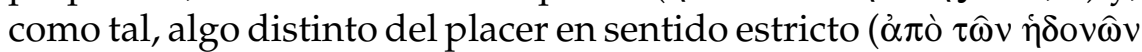

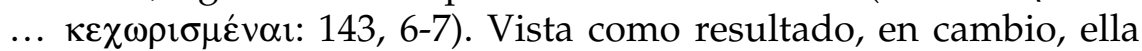
es causa formal del mismo y se identifica con su esencia, que

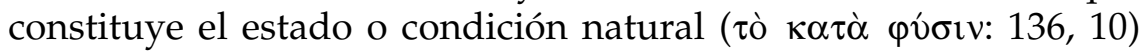

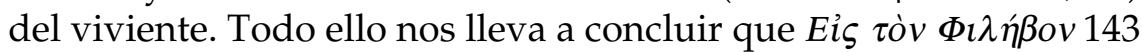
y 136 de Damascio es una lectura fiel de Filebo 31d-e.

Teniendo en cuenta la idea de "estado natural", presente en ambos loci, comento en mi libro ${ }^{27}$ que "un viviente sin placer es, desde el punto de vista fisiológico, un aborto de la naturaleza". Es una afirmación coherente con la que Van Riel (p. 300) lee en Filebo 21d-e y 32d-33c: "una vida sin placer nunca puede ser la vida buena". Pero se opone a la que el mismo Van Riel concluye un poco más adelante: "en el estado natural, el placer está ausente per definitionem" (O.c., p. 301). ¿Quiere esto decir que "vida buena" y "estado natural" se oponen entre sí? Prefiero pensar que la oposición se da sólo entre la doctrina del Filebo y la lectura de Van Riel. Según éste autor, la $\pi \lambda \eta ́ p \omega \sigma ı \varsigma$ de la definición fisiológica del placer es siempre proceso. El placer existe mientras el proceso dura; cuando éste termina y se restablece el "estado de naturaleza", el placer desaparece. Uno tiene la impresión de estar ante Calicles y sus toneles agujereados: "el hombre con los toneles llenos -sostiene

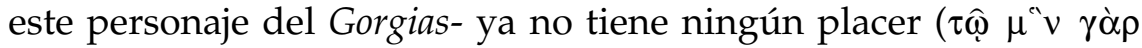

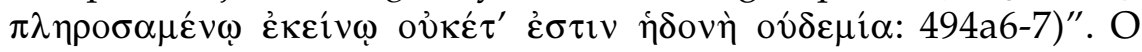
aún más explícitamente: "una vez llenados los toneles, ya no se

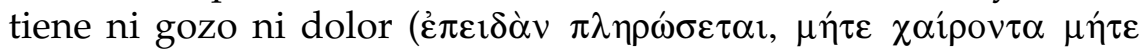

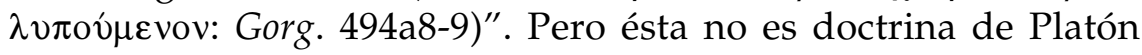
sino de Calicles, quien, adoptando un hedonismo sibarita, sostiene

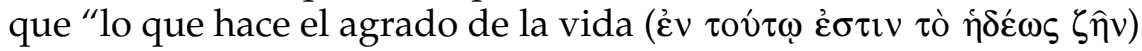
es verter [en la copa del goce] lo más que se pueda ( $\dot{\varepsilon} \nu \tau \hat{\omega} \omega \hat{\omega} \varsigma \lambda \lambda \varepsilon \hat{\imath} \sigma \tau$ ov

${ }^{27}$ F. Bravo, Las ambigüedades del Placer. Ensayo sobre el placer en la filosofía de Platón, Sank Augustin, Academia Verlag, 2003, p. 59.

Educ. e Filos., Uberlândia, v. 22, n. 43, p. 139-160, jan./jun. 2008. 


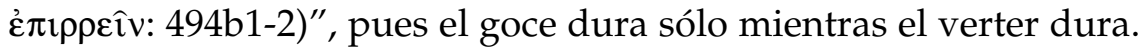
Es lo que exige un placer que es sólo proceso. Platón cree, por el contrario, que tener que llenarse sin tregua no es propio de una

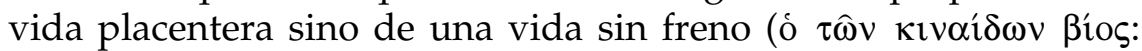
494e4-5), y que una vida de esta índole es "espantosa, vergonzosa

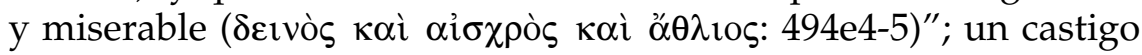
de los dioses, que, según el mito, han condenado a los injustos a

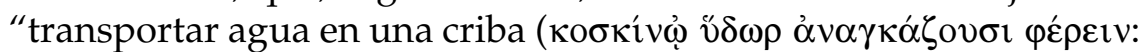
Rep. 363d8)".

2. George Rudebusch, en su estimulante análisis de mi libro Las ambigüedades del placer $^{28}$, sostiene que la lectura de Damascio y la mía tienen por objeto "asimilar la explicación platónica del placer a las sofisticadas y plausibles explicaciones de Aristóteles y Epicuro". Debo confesar que mi objetivo no ha sido esta asimilación, sino la intelección de la doctrina platónica, ciñéndome, en lo posible, a la letra y al espíritu de los textos. Quisiera, sin em,bargo, tratar de responder a sus sutiles objeciones.

I. Objeciones desde el punto de vista de la "fidelidad textual".

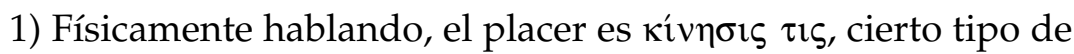
movimiento. Pero, objeta Rudebusch, un movimiento no es un estado... No, si se trata del movimiento físico, que necesariamente tiene lugar en el espacio y requiere un aquí y un ahí. Pero el placer es un movimiento en tanto que es "generado en el alma" ( $\dot{\varepsilon} v \psi v \chi \hat{n}$

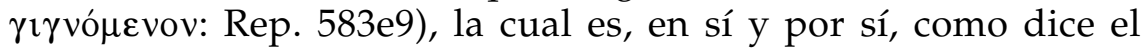

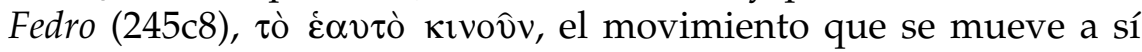
mismo $^{29}$. Es, pues, un movimiento del alma (cf. Fil. 35d1-2) ${ }^{30}$ o movimiento psíquico. Como tal: (1) es, como lo sugiere Cratilo 411a413d, un movimiento no-espacial, semejante al de la sabiduría

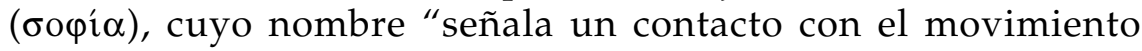

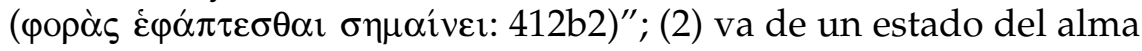

${ }^{28}$ G. Rudebusch, Ancient Philosophy 26 2006).

${ }^{29}$ Cf. LAP, p. 50.

${ }^{30}$ Cf. Bravo (2003) 86, 92. 


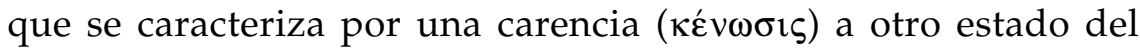
alma que se define como plenitud ( $\pi \lambda \eta \dot{\eta} \omega \sigma \iota \varsigma)$. Y al alcanzar este segundo estado, lejos de desaparecer como movimiento, deviene

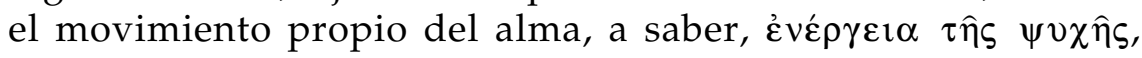
actividad del alma. Y esto vale tanto para los placeres mentales como para los placeres somáticos, pues, independientemente de su ubicación específica, tienen como sujeto último al alma.

2) Según Rudebusch, Platón niega que el estado de repleción que sigue al proceso de llenar sea un estado neutro. Lo niega efectivamente. Para el Filebo, la repleción-estado no es un estado neutro, que se caracterizaría por la ausencia de placer y dolor, sino un estado de placer, que es el estado natural del viviente.

3) La principal objeción de este grupo va dirigida contra mi traducción de $\dot{\alpha} \rho \mu \mathrm{\tau} \tau \tau$ 31d8. Esta traducción -objeta Rudebusch- supone erróneamente que $\alpha \rho \mu о \tau \tau o \mu \varepsilon \dot{\varepsilon} \nu \eta \varsigma$ es un participio pasivo perfecto (es decir,

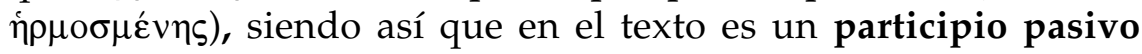
presente. No se refiere, pues, a 'la armonía recuperada', sino 'la armonía que está siendo recuperada'. Ahora bien, objeta Rudebusch, Platón emplea siempre el participio pasivo presente para referirse a un proceso (cita Rep. 349d y 591d) y el participio pasivo perfecto para referirse a un estado (cita Rep. 410e, 443e y 554e, Fedón 93d y Laques 188d). Por tanto, el participio pasivo presente que usa Filebo $31 \mathrm{~d} 8$ no apoya, sino que refuta la interpretación de $\pi \lambda \eta \dot{\eta} \omega \sigma$ เৎ como un estado. Es, a primera vista, una objeción demoledora. Una objeción que también puede dirigirse a Damascio y a algunos traductores modernos. No a mi traducción, sin embargo, pues "la armonía es recuperada" que yo propongo no es traducción del participio pasivo perfecto

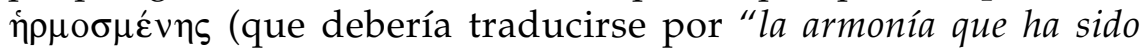
recuperada"), sino del participio pasivo presente, pues equivale a "la armonía que está siendo recuperada". ¿Puede, empero, ser armonía, una que no pasa de estar siendo recuperada? Claro que esta pregunta va más allá de la lingüística, y debo reconocer que mis razones para defender el sentido pasivo (o estático) de ' $\pi \lambda \eta ́ p \omega \sigma r \varsigma^{\prime}$ no son sólo ni sobre todo de carácter lingüístico -que

Educ. e Filos., Uberlândia, v. 22, n. 43, p. 139-160, jan./jun. 2008. 
son más importantes para los filósofos analíticos que para el autor del Filebo- sino de índole teórica. Insisto, pese a ello -apoyándome en algunos helenistas reconocidos (Bally, Liddel-Scott, etc.) ${ }^{31}{ }_{-}$, que, en el griego clásico ordinario, el término ' $\pi \lambda \eta \dot{\eta} \omega \sigma \iota \varsigma^{\prime}$ tiene tanto el sentido activo de repleción como el pasivo de plenitud. No descuidaré, sin embargo, las sabias observaciones lingüísticas de mi objetor, alegando muy cómodamente que Platón no se caracteriza por la precisión semántica.

II. Pero las objeciones de Rudebusch también atañen al campo teórico.

(1) Me atribuye el "deseo" de asimilar la definición platónica del placer a la aristotélica de EN VII, 12, 1153a13-15, que, según él, es un rechazo de aquélla. Para Aristóteles, "no es exacto decir

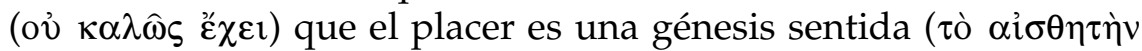
$\gamma \varepsilon ́ v \varepsilon \sigma \iota v)$, sino que es mejor definirlo como una actividad del estado

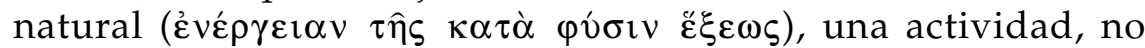

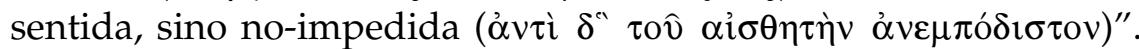
Insisto en que el móvil de mi análisis no ha sido el "deseo" de asimilar la definición platónica a la aristotélica. Lo que ha ocurrido es que, tras encontrar en Platón tanto el sentido activo como el sentido pasivo de 'repleción', he concluido: (a) que el segundo no está lejos de la definición aristotélica; (b) que ésta definición ha sido en cierto modo preparada por la idea de repleción-estado presente en el Filebo. En efecto, también el placer concebido como repleción-estado, privilegiada por el neoplatónico Damascio, es,

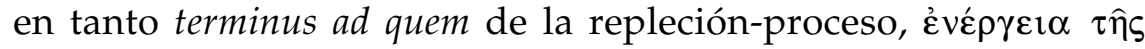
$\psi v \chi \hat{\eta} \varsigma$, actividad del alma, y, como tal, tan floración del estado

${ }^{31}$ Bally (p. 1573) distingue una $\pi \lambda \eta ́ p \omega \sigma \iota \varsigma$ transitiva, que denota la "action de remplir" (Fil. 42c), y otra intransitiva, que designa "l'état de ce qui est plein ou complet, plénitude". Para Liddell-Scott (p. 1420), $\pi \lambda \eta \dot{n} \rho \omega \sigma \iota \varsigma$ significa: 1. filling up (Fil. 42c, Leyes 956e); 2. sensual satisfaction, gratification (Gorg. 496e, 492a; Rep. 439d; Fil. 31e ss, 35a ss).

Educ. e Filos., Uberlândia, v. 22, n. 43, p. 139-160, jan./jun. 2008. 


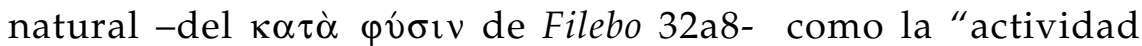
perfecta" de EN X o "la actividad no impedida" de EN VII ${ }^{32}$.

(2) Según Aristóteles, un estado -el de estar sano, por ejemploes teóricamente insuficiente para constituir un placer. Para que

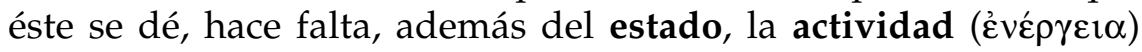
que le es propia, $\mathrm{y}$, los estados ( $\xi^{\xi} \xi \varepsilon 1 \varsigma$ ) qua tales no comportan actividades $^{33}$. Los "meros estados", ciertamente no; pero sí los "estados sentidos" o conscientes, como es el caso del placer. Según Filebo 51b6, los placeres estéticos, por ejemplo, son $\pi \lambda \eta \rho \omega \sigma \sigma \varepsilon ı s$ $\alpha i \sigma \theta \varepsilon \tau \alpha i$, plenitudes sentidas. Timeo 64d2 extiende el atributo de 'sentidos' a los otros tipos del placer ${ }^{34}$, precisando las condiciones para que lo sean. No se puede, pues, asimilar el placer-estado al tipo de estado ( $\varepsilon \xi 1 \varsigma)$ que es la virtud, la cual puede dejar de ser una actividad ( $\dot{\varepsilon} v \varepsilon \hat{\varepsilon} \rho \varepsilon \imath \alpha)$ e hibernar como simple capacidad

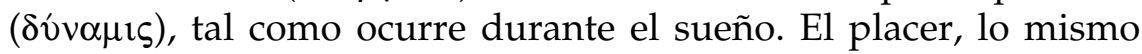

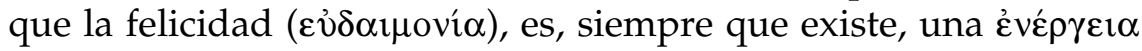
$\tau \hat{\eta} \varsigma \psi v \chi \hat{\eta} \varsigma$, y ello porque es, siempre que existe, una "repleción

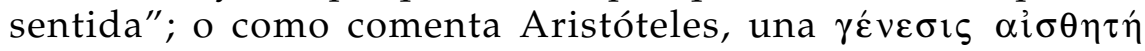
(1153a13). Si Aristóteles no asimila la definición platónica a la suya no es porque Platón concibe el placer como una repleción activa y

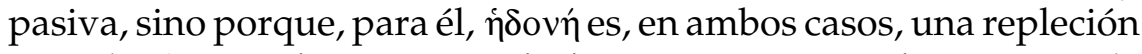
sentida. Según el Estagirita, el placer no es una repleción sentida ( $\alpha i \sigma \theta \eta \tau \eta ́)$, sino una repleción no-impedida ( $\alpha v \varepsilon \mu \pi o ́ \delta ı \sigma \tau \nu)$.

(3) ¿Aceptaría Platón que el placer es una repleción (activa o pasiva) no impedida, como lo define Aristóteles? Rudebusch cree que no. Sospecha, en efecto, que en Filebo 33b, Sócrates "appears to deny that the unimpeded activity of intellect itself is a pleasure". Según su lectura de este texto: (a) "no es improbable ('i $\sigma \omega \varsigma$ ov́ $\delta$ " $v$ ö $\tau 0 \pi \circ v)$ " que los dioses activen su intelecto y lo hagan sin ningún impedimento; (b) sin embargo, no sienten ningún placer. Mi lectura es un tanto diferente. Según ella: (a) "no es improbable" que "la vida de inteligencia y sabiduría", en la que no se experimenta

${ }^{32}$ Cf. Bravo (2003) 71.

${ }^{33}$ Ética a Nicómaco, I, 1, 1095 b 30 - 1096 a 2 y X, 6, 1176 a 33 - b1.

${ }^{34}$ Bravo (2003) 84.

Educ. e Filos., Uberlândia, v. 22, n. 43, p. 139-160, jan./jun. 2008. 
placer alguno, sea "la más divina de todas"; (b) por tanto, "no es verosímil que los dioses gocen ni experimenten la afección contraria", es decir, el placer. ¿Por qué no lo es? No en virtud de (a), que es meramente probable, sino porque su actividad, pese a ser no-impedida, no cumple con las otras condiciones platónicas del placer: por una parte, no comporta ni puede comportar repleción, que implica un vacío previo; por otra, menos aún comporta repleción sentida, que, como sabemos, implica vida sensorial en aquel que la experimenta. Aristóteles, que rechaza estas dos condiciones y las reemplaza por la de que el placer sea una actividad no-impedida, no tiene dificultad en atribuir a los

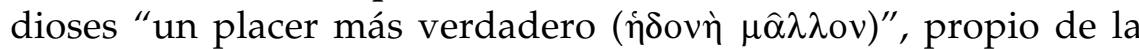

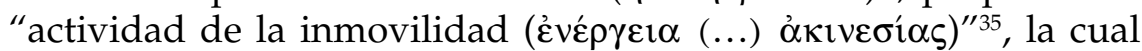

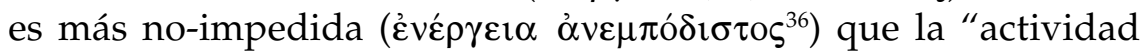

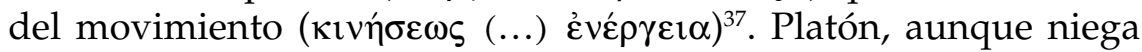
que los dioses experimenten placer, no niega que los placeres verdaderos $\left(\dot{\alpha} \lambda \eta \theta \varepsilon \hat{\imath} \varsigma^{38}\right)$, a saber, los no mezclados ( $\left.\tau \dot{\alpha} \varsigma \dot{\alpha} \mu \varepsilon \hat{\imath} \kappa \tau o v \varsigma^{39}\right)$ o puros $\left(\kappa \alpha \theta \alpha \rho \grave{\varsigma} \varsigma \dot{\eta} \delta o v \alpha ́ \varsigma^{40}\right)$ sean actividades no-impedidas. Más aún, lo afirma implícitamente, pues, por una parte, la repleción definitoria del placer propiamente dicho tiene como resultado fundamental la armonía ( $\left.\alpha \rho \mu \operatorname{vi}^{\prime} \alpha^{41}\right)$ y como contio sine qua non la mesura ( $\left(\dot{\varepsilon} \mu \varepsilon \tau \rho^{\prime} \alpha^{42}\right)$, y por otra, constituye el estado natural ( $\tau$ ò

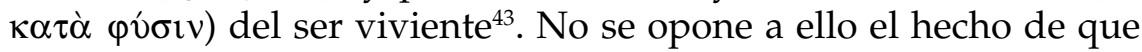
sea una repleción sentida, es decir, consciente. La repleción-estado es, a la vez, sentida y no-impedida. No-impedida, porque es inseparable de la armonía y la mesura ( $\left.\dot{\varepsilon} \mu \varepsilon \tau \rho^{\prime} \alpha\right)$ : "en la medida en que los placeres puros tienen $\dot{\varepsilon} \mu \mu \varepsilon \tau \rho i ́ \alpha$-comenta oportunamente

${ }^{35} E N, 1154$ b $27-28$.

${ }^{36} E N, 1153$ b 11.

${ }^{37}$ Cf. $E N, 1154$ b $26-29$.

${ }^{38}$ Fil. 51 b 1

${ }^{39}$ Fil. 50 e 6.

${ }^{40}$ Fil. 52 c 2.

${ }^{41}$ Fil. 31 d 4, 8; 31 c 11.

${ }^{42}$ Cf. Fil. 52 d 1.

${ }^{43}$ Damascio, 264, 1-3.

Educ. e Filos., Uberlândia, v. 22, n. 43, p. 139-160, jan./jun. 2008 
Gabriela Carone ${ }^{44}$ - tienen $\pi \varepsilon ́ p \alpha \varsigma$ y por tanto estabilidad"; y se remite al marco ontológico de Filebo 24d5, 26d9, 25a6-b3, $26 \mathrm{~b} 2$. Sentida o consciente, y esto de manera inmanente. No requiere, pues, una nueva $\alpha i ̋ \theta \varepsilon \sigma i \varsigma$, que sobrevendría al estado de estar lleno, como insinúa Rudebusch ("nor is there necessarily pleasure in (iv)

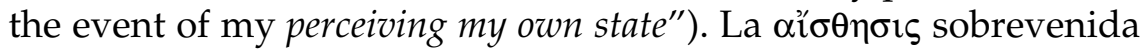
(por ejemplo, la percepción a posteriori de mi estado de buen tejedor)

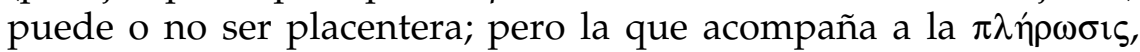
sea ésta activa o pasiva, lo es ex definitione.

(4) Según la interpretación que estoy proponiendo, el placer es, en el plano físico, movimiento, en el plano fisiológico, movimiento de repleción, y en el plano psicológico, movimiento de repleción sentida, o como comenta Aristóteles, $\alpha$ ǐ $\sigma \theta \eta \tau \grave{\eta}$ $\gamma \varepsilon ́ v \varepsilon \sigma i \varsigma^{45}$, proceso consciente. Rudebusch objeta que el tránsito del nivel fisiológico al psicológico "no contribuye a que la interpretación pasiva de $\pi \lambda \eta ́ \rho \omega \sigma \iota \varsigma$ alcance suficiencia teórica". Creo, por el contrario, que sí la ayuda y que es coherente con ella. $\mathrm{Al}$ ser una repleción sentida, es eo ipso una repleción consciente ${ }^{46}$ $\mathrm{y}$, qua talis, una repleción atenta o dotada de la atención del sujeto. G. Ryle señala oportunamente que, "para una persona cualquiera, es imposible, no psicológica sino lógicamente, estar gozando de la música sin poner ninguna atención en ella" ${ }^{\prime 7}$. Ahora bien, si el placer es una repleción atenta es, por el mismo hecho, una disposición del sujeto y no un mero episodio que le afecta desde fuera. Siendo una disposición desde el punto de vista psicológico, puede ser también, sin contradicción, tanto repleción-proceso como repleción-estado ${ }^{48}$.

Recordemos, para terminar, que la tesis del placer-génesis, teóricamente próxima a la concepción del placer como repleción-

${ }^{44}$ G. Carone, "Hedonism and the pleasureless life in Plato's Philebus", Phronesis $45 / 4(2000) 268$.

${ }^{45} E N, 1153$ a 13.

${ }^{46}$ Recordemos que 'consciente' es una de las posibles acepciones de ' $\alpha i \sigma \theta \eta \tau \eta '$ '.

${ }^{47}$ G. Ryle, "Pleasure, Dilemas, Cambridge, At the U. Press, 1954, pp. 58-59; cf. Bravo (2003) 107.

${ }^{48}$ Cf. Bravo (2003) 105-106.

Educ. e Filos., Uberlândia, v. 22, n. 43, p. 139-160, jan./jun. 2008. 
proceso, aunque es favorablemente acogida por Platón en vista de

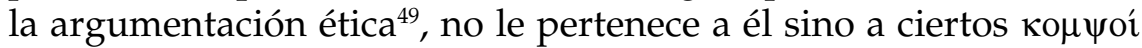
de su época. Recordemos, además, que los "mixtos" del Filebo, compuestos de Límite e Ilimitado, no son puras $\gamma \varepsilon v \varepsilon ́ \sigma \varepsilon 1 \varsigma$, como pretenden los "sutiles" con respecto al placer, sino, en una primera

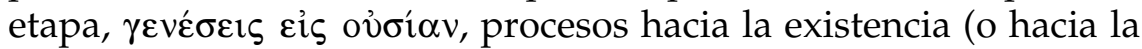
esencia) y, en una segunda etapa, en tanto productos del proceso

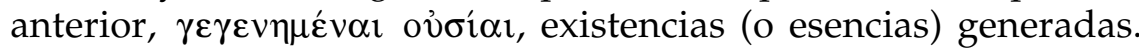
Ahora bien, es doctrina del Filebo que el placer pertenece, en el nivel ontológico, al género de los "mixtos" $\gamma \varepsilon \dot{v} \varepsilon \sigma i s$, en tanto repleción-proceso, sino también una $\gamma \varepsilon \gamma \varepsilon v \eta \mu \varepsilon ́ v \eta$ ov̉ $\sigma i \alpha$, una existencia (o esencia) generada, en tanto repleciónestado $^{51}$. Como tal, entra a formar parte de la vida buena, que no

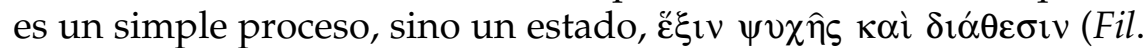
11d4), un hábito y una condición del alma.

\section{BIBLIOGRAFÍA}

A. Bailly, Dictionnaire Grec-Francais, Paris, Hachette, $1950=$ Bailly (1950).

Bravo, Las ambigüedades del placer. Ensayo sobre el placer en la filosofía de Platón, Sankt Augustin, Academia Verlag, 2003 = Bravo (2003).

Carone, G., "Hedonism and the pleasureless life in Plato's Philebus", Phronesis 45/4 (2000).

Liddell, H.G. \& R. Scott, A Greek-English Lexicon, Oxford, At the Clarendon Press, 1968 = Liddell-Scott (1968).

Maudsley, Phisiology and Pathology of Mind, 1867 = Maudsley (1867).

${ }^{49}$ Fil. 53 c.

${ }^{50}$ Cf. Fil. 31 c 2.

${ }^{51}$ Cf. Bravo (2003) 62. 
Rudebusch, G., Review F. Bravo, Las ambigüedades del placer, Sankt Augustin, Academia Verlag, 2003, in: Ancient Philosophy 26 (2006).

Ryle, G., "Pleasure", Dilemas, Cambridge, At the University Press, 1954 = Ryle (1954).

Taylor, A.E., Commentary on Plato's Timaeus, Oxford, At the Clarendon Press, $1928=$ Taylor (1928).

Van Riel, G., "Le plaisir est-il la répletion d'un manque? La définition du plaisir (32a-36c) et la physiologie des plaisirs faux (42c-44e), in: M. Dixsaut, La fêlure du plaisir, Paris, J. Vrin, $1999=$ Van Riel (1999).

Westerink, G., Lectures on the Philebus wrongly attributed to Olympiodorus, Amsterdam, North Holland, 1959. 